Hussain A Obaidi

BDS, MSc, (Prof)

\section{Omar Sabah}

BDS, MSc (Assist Lect)

Evaluation of Tipping, Rotation and rate of space Closure Rate of Canine Retraction by friction less orthodontic techniques (An In vitro Study).

\author{
Dept of Pedod, orthod, and Prev Dentistry \\ College of Dentistry, University of Mosul \\ Dept of Pedod, orthod, and Prev Dentistry \\ College of Dentistry, University of Mosul
}

\title{
ABSTRACT
}

Aim: To evaluate the effect of loops geometry (design) on the rate of space closure, degree of rotation, and degree of tipping. Materials and Methods: The sample consisted of 8 type of loops, namely vertical-loop, vertical with helix-loop, T-loop, readymade loop, PG (universal retraction spring)-loop, opus-loop, teardrop-loop, and L-loop. The method adapted in present study was carried out on Typodont simulation system. The data subjected to the descriptive and variance statistics at 0.05 significant level. Results: There was a significant difference between loops in rate of space closure, rotation and tipping. Vertical loops gave higher significance of rate of space closure and also higher significance degree of rotation and tipping when compared with other loops, while the readymade loop is lowest significance a rate of space closure but it gave low degree rotation and tipping. Although Tloop gave a rate of space closure less than vertical loops but it had the lowest significant degree of rotation and tipping. Conclusion: The T-loop is so more controls and simplest than other loops performing low significance of rotation and tipping tooth movements and achieving higher significance in rate of space closure,

Key words: Rate of space closure, Degree of rotation, Degree of tipping.

Obaidi HA, Sabah O. Evaluation of Tipping, Rotation and rate of space Closure Rate of Canine Retraction by friction less orthodontic techniques (An In vitro Study). Al-Rafidain Dent J. 2006; 6(Sp Iss ): $30 \mathrm{~S}-37 \mathrm{~S}$.

Received: Sent to Referees: Accepted for Publication:

\section{INTRODUCTION}

Over the years, orthodontics have increasingly shown more interest in the problem of retraction of permanent canine. This tooth movement is necessary in space closure of the arch for resolving different orthodontic problem. Very often, the extraction of the first bicuspid is necessary in case where there is sever crowding due to discrepancy in tooth size and jaw size. ${ }^{(1)}$ Mckee et al., (2) reported that the major objective of orthodontic treatment is to correct tooth in 3-plane of space with the forces and moments necessary for this controlled tooth movement. Fixed appliances are necessary to transmit the necessary forces and moments to the teeth. ${ }^{(3)}$

Andrews ${ }^{(4)}$ and Reha et al., ${ }^{(5)}$ found that, precise control of tooth movement during closure of extraction spaces in three dimensions is of paramount importance in meeting treatment goals.
Both friction (sliding) and frictionless (loop) mechanics are used for space closure in extraction therapy. In sliding mechanics, the wire and position of the bracket give control of tooth movement, whereas in a loop-spring system, control is built into the spring ${ }^{(6)}$, but friction binding remains the main problem associated with sliding source of force. ${ }^{(7)}$

One of the major advantages of frictionless mechanics is that a known force system is delivered to teeth because there is no dissipation of force by friction. ${ }^{(6)}$ So the using of sectional arch mechanics (frictionless) method for canine retraction with different closing loops can overcome such a problem. ${ }^{(7)}$

The present study is concerned with canine retraction with different sectional closing loops mechanics; vertical-loop, vertical with helix-loop, T-loop, readymade loop, PG (universal retraction spring)loop, opus-loop, teardrop-loop, and L-lo- 
op, to evaluate the control of tipping and rotation and rate space closure during canine retraction.

\section{MATERIALS AND METHODS}

The sample consisted of 8 type of loops, namely Gjessing Closing Loop ${ }^{(8,9)}$ (GL), Opus Loop ${ }^{(10)}(\mathrm{OL})$, PG Loop ${ }^{(9)}$ (PG), readymade loop ${ }^{(11)}$ (RML) (Dentarurum-Germany), T-Loop'10 (TL); Teardrop Closing Loop ${ }^{(11)}$ (TDL); L-Loop ${ }^{(11,10)}$ (LL), Vertical Loop ${ }^{(10)}$ (VL); Vertical Loop with Helix ${ }^{(11)}$ (VHL) (Figure 1).

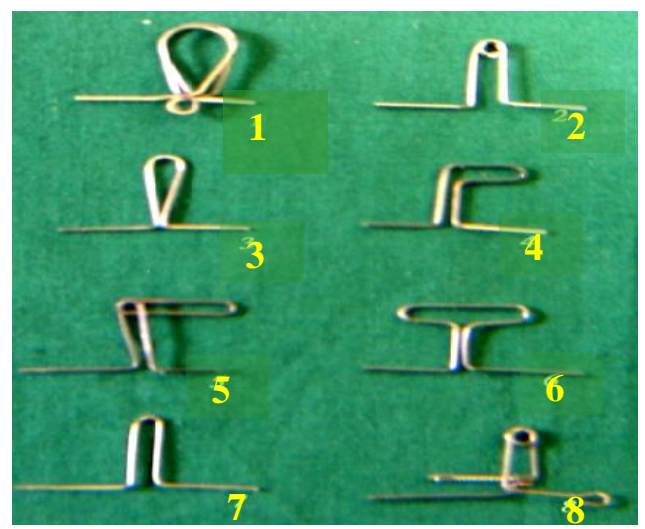

Figure (1): Eight types of sectional closing loops; (1): Readymade- loop; (2): Vertical with helix-loop; (3): Teardrop-loop; (4): L-loop; (5): Opusloop; (6): T-loop; (7): Vertical-loop; (8): Universal retraction- loop.

The method include: Banding theCL II division 1 malocclusion Typodont teeth (CL II division 1) malocclusion (OrmcoJapan) and in approximately well aligned position, Precise final alignment for these teeth were done, with S.S. rectangular arch wire of size $(0.018$ " x 0.025 " DentarurumGermany).

Construction of acrylic bite plane which must be extended in such away that all the (incisal and occlusal third of facial surfaces), (incisal edges and occlusal surfaces of all Typodont teeth), (distal aspect of the lateral incisors), (mesial aspect of the second premolars), palatal surfaces and till the distal extension of the Typodont base; Two small screws made through the acrylic bite plane to be tightened into the metalic base of the Typodont, the distance between these two screws is $(2 \mathrm{~cm})$. These two points aid in immobilization of Typodont teeth while movement of canine was happened.

Immobilization measuring bars; an (Lshape) bar made from S.S. rectangular wire of size $\left(0.018 \times 0.025^{\prime \prime}\right)$, the short arm was welded to distal aspect of canine's band that's extend upward incisally for (10 $\mathrm{mm}$ ) distance then it bends at right angle to extend anteriorlly $(20 \mathrm{~mm})$, and $(5 \mathrm{~mm})$ over canine cusp tip and under the bite plane extension bar by about $(5 \mathrm{~mm})$, an (Lshape) bar made from S.S. rectangular wire of size $(0.018 \times 0.025 ")$, the short arm was inserted in a groove made in the simulated rugae area of the acrylic bite, and then cold cure acrylic was painted over the bar's part that's placed in the groove to make it immobile. This bar emerges upward for $(10 \mathrm{~mm})$ distance then it bends at right angle to extend facially $(20 \mathrm{~mm})$ in a canine direction making right angle with (canine extension bar) from horizontal and vertical direction.

These two bars were used as a guide for determining position, degree of tipping and rotation of canine following sliding movement. This method is a modification of Huffman and Way ${ }^{(12)}$ procedure for determining position, degree of tipping and rotation of the canine following sliding movement.

Rate of Canine Movement (Space Closure) Measurement: First the extracted space (space available) was measured and then the rate of space closure was measured after each method of canine movement, where again the distance between the distal wing of canine's bracket and the mesial wing of second premolar's bracket was measured $^{(13)}$ using Digital Vernia. This distance was considered as the remaining space, therefore:

Rate of Space Closure = Available Spaceemaining Space.

Degree of canine's tipping after movement: Measurement was made according to Ziegler and Ingevall method ${ }^{(14)}$ with so-me modifications that's simplified the method. A Typodont was photographed with transverse projection from right side of Typodont, directly toward right canine where the angle is exposed and then can be measured directly on the photograph, this angle was considered as canine's bar inclination angle, therefore: Degree of 
Canine's Tipping $=$ Canine's Bar Original Angle - Canine's Bar Inclination Angle.

Degree of Canine's Rotation After Movement: Measurement was also made according to Ziegler and Ingevall method ${ }^{(14)}$ with some modifications that's simplified the method. A Typodont was photographed with vertical projection from occlusal side of Typodont, directly toward right canine where the angle is exposed and then can be measured directly on the photograph; this angle is considered the canine's rotation angle, therefore: Degree of Cani- ne's Rotation $=$ Canine's Bar Original Angle - Canine's Bar Rotation Angle.

\section{RESULTS}

Rate of Space Closure: Tables $(1,2)$ showed that the V.L. has insignificant increase in rate of space closure than the V.H.L. and TD.L. While V.H.L. has insignificant increase in rate of space closure than the; TDL , O.L., L.L, and PG.L. The RML . has significantly decreased in rate of space closure than all other investigated loops.

Table (1): Descriptive Analysis of space closure

\begin{tabular}{cccccccc}
\hline $\begin{array}{c}\text { Loops } \\
\text { Code }\end{array}$ & $\begin{array}{c}\text { Type of } \\
\text { Loop }\end{array}$ & Number & Mean* & SD & SE & Minimum & Maximum \\
\hline $\mathbf{1}$ & V.L & 10 & 0.81 & 0.08 & 0.03 & 0.74 & 1 \\
$\mathbf{2}$ & V.H.L & 10 & 0.772 & 0.1 & 0.033 & 0.7 & 0.99 \\
$\mathbf{3}$ & TD.L & 10 & 0.747 & 0.09 & 0.03 & 0.5 & 0.82 \\
$\mathbf{4}$ & O.L & 10 & 0.734 & 0.05 & 0.02 & 0.62 & 0.81 \\
$\mathbf{5}$ & T.L & 10 & 0.711 & 0.1 & 0.03 & 0.57 & 0.85 \\
$\mathbf{6}$ & L.L & 10 & 0.708 & 0.04 & 0.01 & 0.67 & 0.77 \\
$\mathbf{7}$ & R.M.L & 10 & 0.618 & 0.04 & 0.02 & 0.57 & 0.69 \\
$\mathbf{8}$ & PG.L & 10 & 0.708 & 0.09 & 0.03 & 0.6 & 0.86 \\
\hline
\end{tabular}

Mean was given in millimeter. Anova test : F value 5.316 and P <0.001. R.M.L:Readymadeloop O.L: Opus-loop. T.L: T-loop. PG.L: Universal retraction-loop. L.L: L-loop TD.L: Teardrop-loop. V.L: Vertical-loop. V.H.L: Vertical with helix-loop

Table (2): Duncan's multiple rang test of Rate of Space Closure.

\begin{tabular}{ccc}
\hline Type of loop & Mean & Duncan groups \\
\hline 1. Vertical-loop (V.L.) & 0.810 & $\mathrm{C}$ \\
2. Vertical with helix-loop (V.H.L.) & 0.772 & $\mathrm{BC}$ \\
3. Teardrop-loop ( TD.L) & 0.747 & $\mathrm{BC}$ \\
4. Opus-loop (O.L) & 0.734 & $\mathrm{~B}$ \\
5. T-loop (T.L.) & 0.711 & $\mathrm{~B}$ \\
6. L-loop (L.L.) & 0.708 & $\mathrm{~B}$ \\
7. Readymade-loop (R.M.L.) & 0.618 & $\mathrm{~A}$ \\
8. Universal retraction-loop (PG.L.) & 0.708 & $\mathrm{~B}$ \\
\hline $\begin{array}{l}\text { The mean was in degree measurement. Different letters mean significantly } \\
\text { different at P } \leq \text { 0.05. }\end{array}$
\end{tabular}


Degree of Rotation: Tables $(3,4)$ showed that V.L has a significantly higher degree of rotation than all other loops. The V.H.L. had on insignificant increase in degree of rotation than TD.L. and significant increase than PG.L, L.L,O.L,T.L, and R.M.L. The TD.L. has insignificant increase than PG.L. and R.M.L. and significant decrease than L.L., O.L. and T.L. the L.L. had sign- ificant more degree of rotation than O.L. and T.L. and a significant decrease than other investigated loops. the O.L. has a significantly increase in degree of rotateon than T.L. and a significant decrease than other tested loops, and T.L. has signifycant decrease in degree of rotation than all studied loops.

Table (3): Descriptive Analysis Degree of Rotation.

\begin{tabular}{cccccccc}
\hline $\begin{array}{c}\text { Loops } \\
\text { Code }\end{array}$ & $\begin{array}{c}\text { Type of } \\
\text { Loop }\end{array}$ & Number & Mean & SD & SE & Minimum & Maximum \\
\hline $\mathbf{1}$ & V.H.L & 10 & 10.5 & 1.69 & 0.53 & 8 & 12 \\
$\mathbf{2}$ & PG.L & 10 & 8.2 & 1.4 & 0.44 & 6 & 11 \\
$\mathbf{3}$ & TD.L & 10 & 9.35 & 1.06 & 0.33 & 8 & 11 \\
$\mathbf{4}$ & L.L & 10 & 6 & 2.06 & 0.65 & 3 & 11 \\
$\mathbf{5}$ & V.L. & 10 & 15 & 2.45 & 0.78 & 12 & 18 \\
$\mathbf{6}$ & O.L & 10 & 4.6 & 0.84 & 0.27 & 4 & 6 \\
$\mathbf{7}$ & T.L & 10 & 2.75 & 0.43 & 0.13 & 2 & 3 \\
$\mathbf{8}$ & R.M.L & 10 & 8 & 1.33 & 0.42 & 6 & 10 \\
\hline
\end{tabular}

Mean was given in degree; R.M.L: Readymade- loop; O.L: Opus-loop; T.L: T-loop; PG.L: Universal retraction-loop; L.L: L-loop; TD.L: Teardrop-loop; V.L: Verticalloop; V.H.L: Vertical with helix-loop.

Table (4): Duncan's multiple rang test of Degree of Rotation.

\begin{tabular}{ccc}
\hline Type of loop & Mean & Duncan groups \\
\hline 1. Vertical with helix-loop (V.H.L) & 10.50 & $\mathrm{E}$ \\
2. : Universal retraction-loop (PG.L) & 8.20 & $\mathrm{D}$ \\
3. Teardrop-loop (TD.L) & 9.35 & $\mathrm{DE}$ \\
4. : L-loop (L.L) & 6.00 & $\mathrm{C}$ \\
5. Vertical-loop( V.L). & 15.00 & $\mathrm{~F}$ \\
6. : Opus-loop (O.L) & 4.60 & $\mathrm{~B}$ \\
7. T-loop (T.L) & 2.75 & $\mathrm{~A}$ \\
8. Readymade-loop (R.M.L) & 8.00 & $\mathrm{D}$ \\
\hline
\end{tabular}

The mean is in degree measurement. Different letters mean significantly different at $\mathrm{P} \leq 0.05$.

Degree of Tipping: Tables $(5,6)$ showed that the T.L. has insignificantly less degree of tipping than the PG-L, R.M.L., L.L., TD.L. and V.H.L.
The L.L. has insignificant decrease in the degree of tipping than TD.L, V.H.L. and O.L., and the V.L. has a significantly increase than all the investigated loops. 


\section{DISCUSSION}

Rate of Space Closure: The vertical loops have an insignificant increase in rate of space closure than vertical with helix and Teardrop loops and this findings corresponds to the findings by and Proffit ${ }^{(10)}$ who concluded that the addition of the helices has "softened" the appliance so that the force characteristics were reduced. Furthermore, concerning the vertical loop th- ere was significant increase in the rate of space closure than the (opus loop, T-Loop, L-loop , readymade-loop and PG-loop)this result is in agreement with that of, Gjessing ${ }^{(9)}$ concluded that PG.L. designed produces optimal canine retraction in related to vertical loop. This difference may be due to the variation in the investigated approach.

Table (5): Descriptive Analysis Values of Degree of Tipping.

\begin{tabular}{cccccccc}
\hline $\begin{array}{c}\text { Loops } \\
\text { Code }\end{array}$ & $\begin{array}{c}\text { Type of } \\
\text { Loop }\end{array}$ & Number & Mean & SD & SE & Minimum & Maximum \\
\hline $\mathbf{1}$ & PG.L & 10 & 1.3 & 0.55 & 0.17 & 0.5 & 2 \\
$\mathbf{2}$ & V.H.L & 10 & 1.6 & 0.74 & 0.23 & 0.5 & 3 \\
$\mathbf{3}$ & L.L & 10 & 1.4 & 0.64 & 0.2 & 0.5 & 2 \\
$\mathbf{4}$ & O.L & 10 & 2 & 0 & 0 & 2 & 2 \\
$\mathbf{5}$ & V.L & 10 & 4.9 & 0.88 & 0.28 & 4 & 6 \\
$\mathbf{6}$ & R.M.L & 10 & 1.35 & 0.67 & 0.21 & 0.5 & 2 \\
$\mathbf{7}$ & T.L & 10 & 1 & 0.33 & 0.11 & 0.5 & 1.5 \\
$\mathbf{8}$ & TD.L & 10 & 1.5 & 0.58 & 0.18 & 1 & 3 \\
\hline
\end{tabular}

Mean was given in degrees; R.M.L: Readymade-loop; O.L: Opus-loop; T.L: T-loop; PG.L: Universal retraction-loop; L.L: L-loop; TD.L: Teardrop-loop; V.L: Verticalloop; V.H.L: Vertical with helix-loop.

Table (6): Duncan's multiple rang test for Degree of Tipping.

\begin{tabular}{ccc}
\hline Type of loop & Mean & Duncan groups \\
\hline 1. Universal retraction-loop (PG.L) & 1.3 & $\mathrm{~A}$ \\
2. Vertical with helix-loop(V.H.L) & 1.6 & $\mathrm{AB}$ \\
3. L-loop (L.L) & 1.4 & $\mathrm{AB}$ \\
4. Opus-loop (O.L) & 2.0 & $\mathrm{~B}$ \\
5. Vertical-loop (V.L) & 4.9 & $\mathrm{C}$ \\
6. Readymade-loop (R.M.L) & 1.35 & $\mathrm{~A}$ \\
7. T-loop (T.L) & 1.0 & $\mathrm{~A}$ \\
8. Teardrop-loop (TD.L) & 1.5 & $\mathrm{AB}$
\end{tabular}

The mean is in degree measurement. Different letters mean significantly different at $\mathrm{P} \leq 0.05$.

The vertical with helix has an insignificant increase in rate of space closure than T-loop, opus loop, L. Loop, PG-Loop, and readymade loop, this result indicates that the various increase in apical portion and the configuration have no significant influence in rate of space closure this agr- ee with Ødegaard. ${ }^{(15)}$

In this study, it was found that opus loop is insignificantly larger in rate of space closure than T-loop. This result contrast with that of Ødegaard ${ }^{(15)}$ who found that the force magnitude of opus loop was greater than that of $\mathrm{T}$-loop, (the force me- 
an of opus loop creates more space retraction than $\mathrm{T}-\mathrm{loop}$ ).

The readymade loop had a significant decrease in the rate of space closure than the other investigated loop. This could be due to the addition of more apical wire and spring laterally which increase flexibility and decrease amount of force of the loop and achieve a significant decrease in the rate of space closure than other studies loops. This is in accordance with that of Haberstock et al. ${ }^{(16)}$

Degree of Rotation: The vertical loop had significantly higher degree of rotation than all studied loops. This finding was in agreement with finding of Ødegaard ${ }^{(15)}$, who showed that increased mesiodistal width at apex of loops in comparison with standard vertical loop led to an increase flexibility of loop and this would lead to decrease the force and the moment. On the other hand, vertical with helix had an insignificant increase in degree of rotateon than teardrop. This could be related to the different in apical configuration, which had no significant influence on the degree of rotation in spite of the change in the amount of wire between vertical with helix and teardrop loop.

Furthermore, the vertical with helix had a significant increase with other investigated loops. This could be due to that vertical with helix loop had high rotation influence than these loop, this finding agreement with the finding of Faulkner et $a l .,{ }^{(17)}$ who stated that addition of a combination of apical helix and lateral one lead to lower M/F ratio in spite of addition of anti-rotation bend which increase the moment to control the rotation but sometime this angle is insufficient in certain loops. On other hand Gjessing ${ }^{(8,9)}$, Proffit ${ }^{(9)}$ who state that a too low a moment was produce but by placing additional wire apically, as in the T-loop increase the amoment applied. Ødegaare ${ }^{(15)}$ was mentioned that decrease in moment because of different angle of twist (such as in Teardrop loop).

The teardrop loop (TD.L) gave an insignificant increase in the degree of rotation that the PG. loop and readymade loops. This could be due to this change in geometry of apical and present of anti-rotation bends as not make a statistically difference between these loops, in spite givi- ng different value of rotation degree and this with agreement with that of Gjessi$\mathrm{ng}^{(9)}$,but that teardrop loop appeared significant increase in degree of rotation than L-loop, T-loop and opus loop, this result obviously due to the less amount of apical wire material which increase the force and moment force of teardrop loop which create more rotate-on. This result agrees with that of Proffit. ${ }^{(10)}$

The result of present study showed that L-loop had significantly more degree of rotation than opus loop and T-loop and significantly decrease than other tested loops. This finding agrees with that Proffit. ${ }^{(10)}$

The result of this study showed that T-loop has given a better control of tooth rotation, these findings are in agreement with Sander ${ }^{(18)}$ who shows that a typical vertical loop produces too low a moment but by placing additional wire apically, as in the T loop, the magnitude of this moment increases with respect to the force and lead to a better control of tooth position in and this finds when comparison with additional wire falling occlusally completely eliminates the needed moment. In addition, it agrees with Vasquez ${ }^{(19)}$ who showed that T-loops give a low load-deflection curve and would generate more control of rotateon.

Degree of Tipping: The descriptive analysis in the present study shows that generally, the degree of Tipping in all sect-ional closing loops is little in comparison with the degree of rotation and this result corresponds to that of Ziegler and Ingervell. ${ }^{(14)}$

The T-Loop gave the lowest degree of tipping and this finding agreement with Ziegler and Ingervell ${ }^{(14)}$, and vertical loop gave the highest degree of tipping and this finding agrees with Hoenigl et al. ${ }^{(3)}$

The vertical loop had significantly increased in degree the of tipping than all investigated loops. This findings agrees with Faulkner ${ }^{(17)}$ who showed that the addition of the single apical helix has the overall effect of reducing the levels of both the force and the moment for given activation. So the legs of the spring were "gabled" (positioned at an angle to the direction of activetion) to create larger moments. These preactivated springs have a 
highly nonlinear $\mathrm{M} / \mathrm{F}$ ratio as a function of activation and type of spring design. Hence, the changes in $\mathrm{M} / \mathrm{F}$ lead to give such degree of tipping.

The opus loop showed an insignificant increase in the degree of tipping than that vertical with helix ,teardrop loop, and L-loop this result could be due to the difference configuration amount of apical wire and present of anti-tipping bend had no statistical influence on degree of tipping among these sectional loops specially in one activation ,this finding accordance with that of Gjeesing. ${ }^{(8,9)}$ On other hand, the opus loop had significantly difference with (PG-loop, T-loop, readymade and vertical loop) and this may by due to change in an-gle between of vertical portion change the force distribution to retraction canine and with agreement with Proffit. $^{(10)}$

This study showed that PG (universal retraction spring) had an insignificant degree of tipping with $\mathrm{T}$-loop, readymade, L-loop and vertical with helix and good control of tooth movement (tipping). This result is in with agreement with Gjessing. ${ }^{(8,9)}$

Furthermore, the PG-loop had significant difference with opus-loop and vertical loop and this with agreement with $\mathrm{Ge}-$ jessing. ${ }^{(9)}$

The result of this study showed that no significant decrease is between T-loops with readymade loops and this finding, may be due to adding spring. In addition the apical helix in readymade loop lead to decrease amount of force and make them more control of rotation. So the result was near to T-loops. ${ }^{(18)}$

Finally, the T-loop had a significant decrease with opus loop and vertical loop, this is in with agreement with Gejessing. ${ }^{(9)}$

\section{CONCLUSION}

The T-loops is recommended for most control of tooth movement (Tipping, Rotation) and the vertical loop is indicated when high rate of space closure is need.

\section{REFERENCES}

1. Anthony A, Gianelly. Two-stage treatme- nt: an outcomes-based assessment. Orthod. Crainofacial. Res. 2000; 1(1): 3-20.

2. Mckee IW, Philip G, Williamson, Ernest W, Giseon HK E. The accuracy of 4 panoramic units in projection of mesiodistal of tooth angulations. Am J Orthod. 2002; 12: $166-175$

3. Hoenigl K.D, Josef Freudenthaler, Micha-el RM, Hans-Peter Bantleon. A new cent-ered T-loop. Am J Orthod. 1995; 9(5): 113-130.

4. Andrews FL. The S.W.A. syllabus of philosophy and techniques. San Diego: LF Andrews Foundation for Orthodontic. Am J Orthod. 1995; 8(2): 120-125.

5. Reha S, Ki S, Haluk I, Hakan HT, Altug AT. Dentoalveolar distraction osteogenesis for rapid orthodontic canine retraction. J Oral Maxillofac Surg. ; 60: 389-394.

6. Kwangchul Choy, Eung-Kwon Pae, ,Kyung-Ho Kim, Young Chel Park, Charles J. Burstone. Controlled Space Closure with a Statically Determinate Retraction System. Angle Orthod. 2001; 72(3): 191198.

7. Dincer M, Iscan HN. The effects of different sectional arches in canine retraction. Eur J Orthod. 1994; 16(4): 317-323.

8. Gjessing P. Biomechanical design and clinical evaluation of new canine-retraction spring. Am J Orthod. 1985;4(4): 353-362.

9. Gjessing P. A Universal Retraction Spring. Am J Orthod. 1994;5(6): 222-242.

10. Proffit WR. Contemporary orthodontics, $3^{\text {rd }}$ ed; St. Louis, Mosby co. 2000; Pp: 567, 572, 574, 578

11. Renfroe, Earl W. Edgewise. 2000; Pp 65, 66, 90, 144.

12. Huffman DJ, Way DC. A clinical evaluation of tooth movement along arch wires of two different sizes, Am J Orthod Dentofacial Orthop. 1983; 83(6): 453-459.

13. Sonis A. Comparison of NiTi Coil Springs vs. Elastics in Canine Retraction. $J$ Clin Orthod. 1994; 293-295.

14. Ziegler P, Ingervall B. A clinical study of maxillary canine retraction with a retracti-on spring and with sliding mechanics. Am J Orthod Dentofacial Orthop. 1989; 95(2): 99-106.

15. degaard J, Dr.Odont, Meling TM, Meling EM. The effects of loops on the torsional stiffnesses of rectangular wires: An in vitro study. Am J Orthod. 1996; 496-505.

16. Aberstock DL, Faulkner MG, Lipsett 
AW, El-Rayes K. Use of vertical loops in retraction systems PartII. Am J Orthod Dentofacial Orthop. 1991; 8(5): 49-153.

17. Faulkner M., Lipsett AW, El-Rayes, K. and Haberstock, D.L. Use of vertical loops in retraction systems. Am J Orthod Dentofacial Orthop 1991; 10: 328-336.

18. Sander FG. Biomechanical investigation of the hybrid retraction spring. Am J Or- ofac Orthop. 2000; 61(5): 341-351

19. Vasquez M, Calao E, Becerra F, Ossa J, Enriquez C, Fresneda E. Initial stress differences between sliding and sectional mechanics with an endosseous implant as anchorage: a 3-dimensional finite element analysis. Angle Orthod. 2001; 71(4): 247-56. 